\title{
VISCERAL LARVA MIGRANS
}

\author{
BY \\ W. DICKSON and R. C. WOODCOCK \\ From the Bolton District General Hospital, Farnworth, Lancashire
}

(RECEIVED FOR PUBLICATION JULY 15, 1958)

There are many causes of persistent eosinophilia in children, but Perlingiero and György (1947) described a case in which there was also hepatic enlargement, anaemia and fever. Two years later Zuelzer and Apt (1949) described eight similar cases and suggested that they formed a definite syndrome. Since then several more have been reported and it has been shown that this syndrome is frequently caused by invasion of the viscera by the larvae of the dog or cat ascarid. We would like to describe a further case and review the progress that has been made in the study of this syndrome.

\section{Case Report}

Marjorie was admitted to hospital as an urgent case on September 14, 1956, because she had had a severe convulsion.

She was nearly 2 years of age, the third child of healthy parents. Her first year was free from illness and she made good physical progress, sitting up at 6 months of age and standing at 9 months. When she was 11 months old she was allowed to crawl about in her grandmother's back yard and it was then observed that she was a dirt eater. Shortly after her first birthday she developed an acute febrile illness with wheezy respirations. She was treated with penicillin and in about five days the fever subsided but the noisy respirations persisted for about one month. After this illness her disposition began to change; she became irritable, miserable and unfriendly and would speak only to her mother and grandmother. Her appetite became poor and she failed to gain weight. These rather indefinite symptoms were still present when she was admitted to hospital.

No other member of the family had had convulsions but the following additional history was obtained about Marjorie. Since the age of 1 month she had what her mother described as mild fits in which she would go unconscious for a few seconds and then lie limp for about 15 minutes. She was then having about two attacks a week but they had become more frequent recently. When she was 6 months of age she had four major convulsions in three days and since then she has had three major attacks.
There was no family history of allergic disease. The family had a cat and a dog; the dog was killed in an accident the night after Marjorie's admission to hospital.

Examination on admission: she was pale, restless and miserable. Her temperature was $103^{\circ} \mathrm{F}$. The only abnormal findings were injection of the throat, enlargement of the tonsillar glands and a few moist sounds in her lungs. Her liver was enlarged three fingers below the costal margin and it was smooth and firm.

She was thought to have had a febrile convulsion following an upper respiratory infection and further investigations were carried out to find the cause of the enlarged liver.

The results of laboratory investigations were as follows: Hb 8.5 g. $\%$, R.B.C. $4,440,000$ per c.mm., C.I. 0.68; W.B.C. 23,000 per c.mm.; eosinophils, $67 \%$; platelets, 272,000 per c.mm.; B.S.R., $31 \mathrm{~mm}$. in the first hour, $61 \mathrm{~mm}$. in the second hour; serum proteins, $8.6 \mathrm{~g} . \%$; albumin, 4 g. $\%$; globulin, 4.6 g. $\%$; electrophoresis $\gamma$ globulin fraction markedly increased. Liver function tests showed serum alkaline phosphatase, 31 units (K.A.); serum bilirubin, within normal limits; thymol turbidity, 2 units; thymol flocculation, normal; zinc turbidity, 9 units; serum cholesterol, $115 \mathrm{mg}$. \%; urine, no abnormalities; W.R. and Kahn tests negative.

Radiographs of the skull were normal; in those of the chest there was mottling in both upper lobes. A Mantoux test 1:1,000 was negative.

No ova or parasites were seen on repeated examination of the patient's stools, the cat's faeces or the dirt from the back yard.

Iliac marrow puncture showed an eosinophilia but most of the cells were mature and the picture was not that of an eosinophilic leukaemia.

The blood of the father, mother and sibs was tested; the W.B.C. and differential count were normal.

Liver Biopsy. At operation, the liver was enlarged and its appearance most striking. It was studded with firm greyish nodules which varied slightly in size but were all a few millimetres in diameter.

A small, wedge-shaped fragment of liver tissue about $1 \mathrm{~cm}$. in diameter, containing some of the nodules, was removed and fixed in formol saline.

Serial sections of all the tissues were made and they showed inflammatory foci separated by normal liver tissue. In some fields the liver cells had been destroyed 


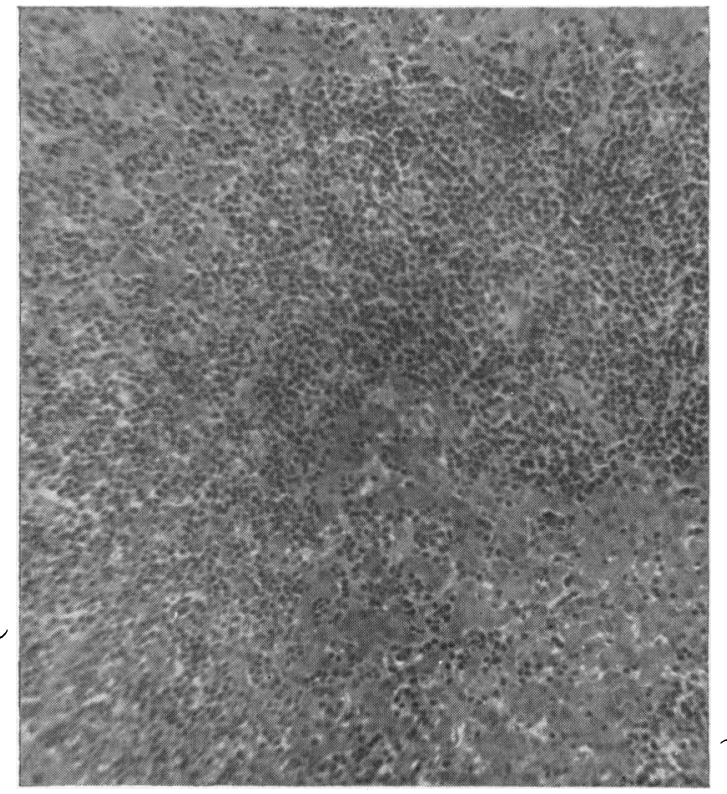

FIG. 1.-Liver. An early lesion. The section shows a focus of inflammatory infiltration consisting mainly of eosinophils. The eosinophils permeate between the liver cells at the periphery of the lesion. In the right hand corner a heavy concentration of eosinophils is shown infiltrating some amorphous debris.

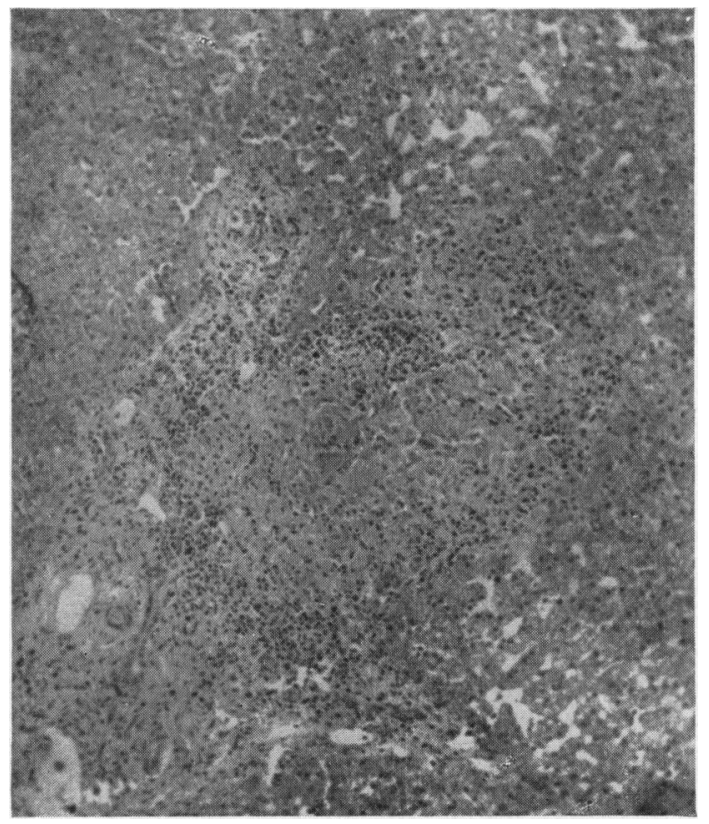

FIG. 2.-Liver. A late lesion-low power. A discrete granuloma consisting of epithelioid cells and giant cells with a zone of lymphocytes and plasma cells surrounding them.

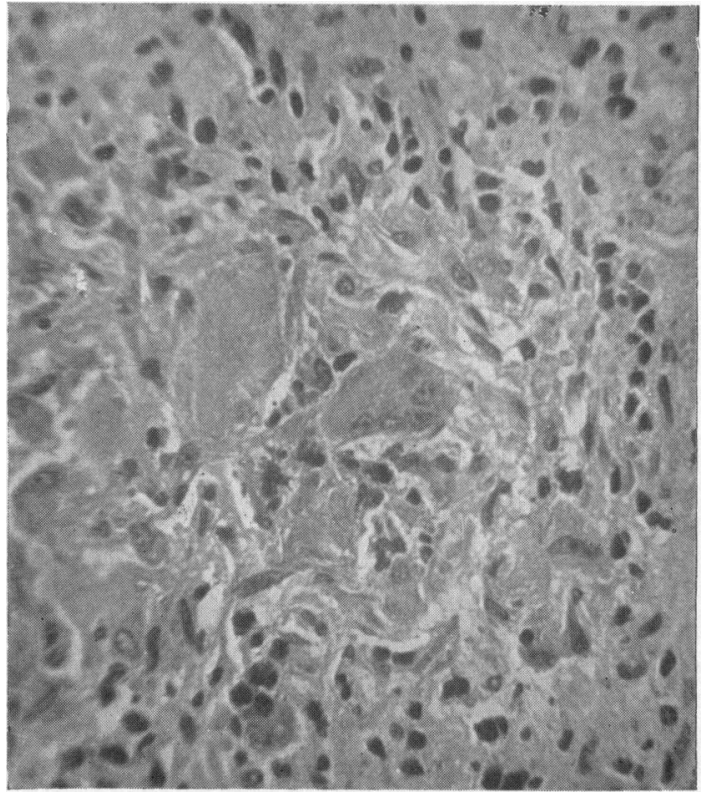

FIG. 3.-Liver. A late lesion-high power. The foreign body giant cells surrounding a clump of amorphous debris are demonstrated.

and replaced almost exclusively by eosinophils. These areas varied in size, were irregular in shape and merged into the surrounding liver tissue. Several distinct clumps of eosinophils invading amorphous débris were seen (Fig. 1) and the appearances strongly suggested that the débris was derived from degenerated fragments of larvae.

There was an inflammatory reaction around and within the portal tracts. In some places most of the cells were eosinophils but in others there was a large proportion of lymphocytes and plasma cells.

Several discrete granulomas were seen. They consisted of ephithelioid cells and giant cells of the foreign body type with a zone of lymphocytes and plasma cells surrounding them (Fig. 2). No foreign body was detected in any of these lesions but some contained fragments of unidentifiable necrotic débris (Fig. 3).

Other similar lesions were undergoing fibrosis and the gritty feel of some of the nodules at operation suggested that calcification may have occurred. This has been reported in the literature in visceral larva migrans.

Jung (personal communication) and his colleagues in New Orleans have examined the sections and agree that the appearances are typical of visceral larva migrans.

Progress. Marjorie has now been observed for nine months and, apart from a course of oral and intramuscular iron, she has had no treatment. The disease has followed a benign course. She has not had any further convulsions or pulmonary symptoms. Her irritability and anorexia have gradually improved and now she is back to normal health. Her eosinophil count 


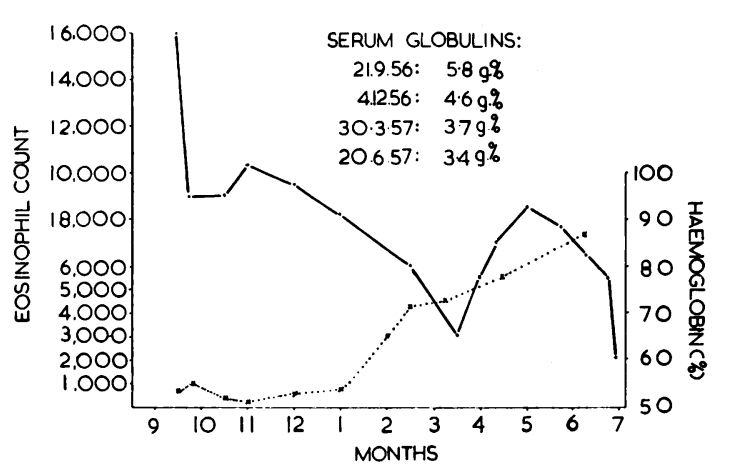

Fig. 4.-Marjorie. Eosinophil count, serum globulins and haemoglobin levels in a case of visceral larva migrans.

has varied but has remained well above normal for more than one year. Her haemoglobin and serum globulin have very slowly returned to more normal levels (Fig. 4).

\section{Review of Literature}

Several reports of a syndrome like the one described have appeared in the American literature. Zuelzer and Apt (1949) were the first to report a group of eight cases which appeared to have a common aetiology and demonstrate a syndrome.

The syndrome occurs in young children and is characterized by pica, pulmonary symptoms with a low grade pyrexia, an enlarged liver studded with eosinophilic granulomas, chronic blood eosinophilia, anaemia and hyperglobulinaemia.

The histology of the liver lesions suggested that the inflammation had an allergic basis but the aetiology was obscure until nine years ago, when Mercer, Lund, Bloomfield and Caldwell (1950) found the larva of an ascarid in a liver lesion. Later Behrer (1951) made a similar finding in another case. They thought that the larvae were those of the human ascarid, but a year later Beaver, Snyder, Carrera, Dent and Lafferty (1952) described three cases and in one a larva was identified as that of the dog ascarid, Toxocara canis. They named the syndrome 'visceral larva migrans' and considered that, like the commoner cutaneous larva migrans, it was due to the migration of nematode larvae through the tissues of an infected person.

A year later Milburn and Ernst (1953) described a further case in which the larva was identified. This case followed the typical benign course, but in the same year Brill, Churg and Beaver (1953) described a fatal case in which granulomas were found in the lungs, heart, liver and kidneys, and the toxocara was again demonstrated. In 1956 Dent, Nichols, Beaver, Carrera and Staggers described the post-mortem findings in a case which died from homologous serum jaundice. Again the characteristic lesions, some containing parasites, were widely scattered in numerous organs, including the brain. It is interesting to note that Beautyman and Woolf (1951) found an encapsulated ascarid in the thalamus of a child dying of poliomyelitis. Further cases have been reported by Heiner and Kevy (1956) and Gault and Webb (1957), writing from South India, described five cases and suggested that invasion of the liver and lungs by nematode larvae is the cause of tropical eosinophilia.

\section{Discussion}

(a) Pathogenesis. The human ascarid, Ascaris lumbricoides, has a rather complicated life cycle. The adult worms live in the small intestine where they feed on its contents and lay eggs. After being discharged in the faeces, the eggs may remain dormant for several years or may embryonate in damp soil in two to four months. Infection is by ingesting the embryonated eggs. The larvae escape from the eggs in the small intestine; they penetrate the wall and are carried to the liver in the portal blood stream. There they rest for a few days before resuming their journey through the right heart to the lungs. During this period they grow considerably in size. After about eight days they are carried up the trachea to the mouth and are swallowed a second time. On this occasion they remain in the bowel and develop to maturity.

The different species of ascarids only complete their life cycles if they are in the correct hosts. The first part of the cycle, that is the larval migration, is not specific. Human ascarids migrate in mice and other animals but none reach sexual maturity. Similarly cat and dog ascarids will migrate in human beings but the larvae will be destroyed in the viscera before they can return to the gut. Thus no eggs will be passed in the faeces as no adult worms are present in the gut. The larvae are destroyed in the viscera of a foreign host and so may not be seen in liver biopsies in all cases. In fact they have been found only in six cases. The inflammatory reaction will be more severe than with the homologous ascarid and thus the signs and symptoms of the disease will be more marked. Beaver et al. (1952) have suggested that patients who develop this syndrome have an allergic diathesis and this may enhance the response to the infection.

Salient Features. PICA. Infection of dogs and cats with toxocara is widespread. The animals may even be infected in utero. Examination of the soil 
from places frequented by them often reveals large numbers of ova.

A history of pica was obtained in over $90 \%$ of cases and ingestion of the ova of the toxocara in the dirt is the source of the infection.

Age Group. The cases belong to a very definite age group: 1 to 3 years.

The reason for this may be that pica is uncommon after 3 years of age or that, in older children, owing to the larger size of the liver capillaries, the toxocara may pass more easily into the lungs. This may account for some of the cases of Loeffler's syndrome, which belongs to an older group and is not associated with hepatomegaly.

Pulmonary Symptoms. Pulmonary symptoms caused by invasion of the lungs by the larvae occur early in the course of the disease and may precede, by a few months, the development of the eosinophilia and hepatomegaly. Common symptoms are cough, coryza and frequently a wheezing type of breathing, often associated with pyrexia. They vary in severity and are often accompanied by radiological changes in the lungs which may resemble miliary tuberculosis. Usually these lung changes are of short duration.

Convulsions. Convulsions and rather obscure cerebral symptoms have been reported in several cases. Zuelzer and Apt (1949) thought that an encephalopathy due to vascular sensitization was possibly part of the syndrome. However, it now appears that infiltration of the brain by larvae may be the cause of the symptoms, for larvae have been found in the brains of mice which have been fed with toxocara ova.

In our case, if the parents' observations are correct regarding the age of onset of the convulsions, it is unlikely that they were caused by the infection. Nevertheless, it is interesting that, as the child's general condition has improved, the convulsions have ceased without treatment.

Hepatomegaly. This is a constant feature in all cases. The liver is usually firm, smooth and regular and may be enlarged as much as five fingers below the costal margin. It seems possible that the parenchymal damage may account for the anorexia, irritability and lassitude. The liver function tests are indicative of parenchymal damage in about $30 \%$ of cases.

ANAEmia. This is also a fairly constant finding. It is of the iron deficiency type but it responds only slowly to oral or intramuscular iron. The average haemoglobin at the height of the symptoms was about $8 \mathrm{~g}$.

Leucocytosis. Leucocytosis with a high percentage of eosinophils was present in all cases at some time in the illness. Counts as high as 120,000 W.B.C. per c.mm. with $87 \%$ eosinophils in the differential count have been recorded, but the average count is about 30,000 W.B.C. per cm. with $50 \%$ eosinophils.

These blood changes may not appear until two to three months after the onset of the respiratory symptoms. Behrer (1951) suggests that the cause of the eosinophilia is the keratin which the larva sheds when it moults in passing through the various stages of its life cycle. Keratin can be demonstrated in liver sections and it is a very powerful eosinophilic stimulant.

HyPerglobulinaemia. A raised serum globulin, due to an increased gamma globulin, has been found in all cases in which it was estimated. This suggests that foreign larvae stimulate a powerful antibody response and that hypersensitivity or serological reactions may prove helpful in diagnosis. This is desirable as the larvae can only be demonstrated with difficulty in liver biopsies, which in children of this age are best obtained by laparotomy. Heiner and Kevy (1956) have' described an agar diffusion precipitation reaction between the patient's serum and an antigen prepared from the toxocara larvae. The test gives promise of providing a specific diagnosis in the near future.

Jung (personal communication) and his associates performed the test on our patient's serum one year after the onset of symptoms. The serum showed no significant titre of antibodies against toxocara or ascaris, so it is possible that this patient may have been infected with a larval worm other than the two mentioned. Jung states that two groups of parasites have been shown in experimental animals to produce symptoms similar to visceral larva migrans: some hookworms and spirurids.

Liver Biopsies. Characteristic lesions have been found in the liver in all cases in which biopsy has been performed. On naked eye examination the presence of numerous small greyish white plaques arranged indiscriminately is strongly suggestive of the diagnosis. Microscopic examination reveals numerous inflammatory foci of varying ages which have been caused by migrating parasites. The younger lesions consist of aggregates, mainly of eosinophils, which have replaced liver cells. The 
more mature ones are granulomas consisting of epithelioid cells and foreign body giant cells surrounded by a zone of lymphocytes and plasma cells. The oldest lesions are granulomas undergoing fibrosis or even calcification.

Larvae are rarely seen in the lesions but small and often degenerate fragments are occasionally found. The very early lesions are most likely to contain a parasite. When they are present the opinion of an expert parasitologist is necessary for their complete identification. The important diagnostic features are as follows: (1) $T$. cati measures $350 \mu$ in length and $17 \mu$ at its greatest diameter. $T$. canis is a little larger. (2) There is a simple oesophagus $120 \mu$ long terminating distally in an elongated bulb $8 \mu$ in diameter, which contains a conspicuous nucleus $3 \mu$ in diameter. (3) In a cross-section larval crests or alae, $1 \mu$ long, may be seen.

Marjorie's progress is typical of the usual course of the disease. The ultimate prognosis is good, although many of the children go through a long period of ill health. No form of treatment has any effect either on the duration of symptoms or the course of the disease.

\section{Summary}

A case of visceral larva migrans is described.

The literature is reviewed and the important signs and symptoms are discussed.
The disease may be an important cause of chronic eosinophilia in childhood and may account for many of the cases considered to be Loeffler's syndrome or tropical eosinophilia.

The disease may be more common in this country than is realized because of the high incidence of toxocara infection in cats and dogs. It is suggested that other parasites of domestic pets, such as hookworms, may produce this condition.

The authors would like to thank Dr. R. C. Jung for examining the serum and sections, Professor W. F. Gaisford for his helpful advice and criticism and Mr. J. Parker. Mr. D. Hart and Miss E. Barton for technical and secretarial assistance.

\section{REFERENCES}

Beautyman, W. and Woolf, A. L. (1951). J. Path. Bact., 63, 635. Beaver, P. C., Snyder, C. H., Carrera, G. M., Dent, J. H. and Lafferty, J. W. (1952). Pediatrics, 9, 7.

Behrer, M. R. (1951). J. Pediat., 38, 635.

Brill, R., Churg, J. and Beaver, P. C. (1953). Amer. J. clin. Path., 23,1208 .

Dent, J. H., Nichols, R. L., Beaver, P. C. Carrera, G. M. and Staggers, R. J. (1956). Amer. J. Path., $32,777$.

Gault, E. W. and Webb, J. K. G. (1957). Lancet, $2,471$.

Heiner, D. C. and Kevy, S. V. (1956). New Engl. J. Med., 254, 629. Jung, R. C. Personal communication.

Mercer, R. D., Lund, H. Z., Bloomfield, R. A. and Caldwell, F. E. (1950) 'Amer. J. Dis. Child., 80, 46.

Milburn, C. L., Jr. and Ernst, K. F. (1953). Pediatrics, 11, 358.

Perlingiero, J. G̈. and György, P. (1947). Amer. J. Dis. Child., 73, 74. Zuelzer, W. W. and Apt, L. (1949). Ibid., 78, 153 